\title{
Neurothekeomas: Dermoscopic Features of 3 Cases
}

\author{
Irene Campana ${ }^{1}$, Elisa Cinotti ${ }^{1}$, Paolo Broganelli ${ }^{2}$, Alessandro Di Stefani ${ }^{3}$, Emanuele Trovato ${ }^{1}$, \\ Luca Provvidenziale ${ }^{1}$, Roberto Perotti ${ }^{1}$, Arianna Lamberti ${ }^{1}$, Pietro Rubegni ${ }^{1}$
}

1 Dermatology Section, Department of Medical Science, Surgery and Neurological Sciences, University of Siena, Siena, Italy
2 A.O.U., Città della Salute e della Scienza di Torino, Turin, Italy
3 Institute of Dermatology, Fondazione Policlinico Universitario A. Gemelli IRCCS, Rome, Italy

Key words: neurothekeomas, dermoscopy, dermatopathology, skin tumors

Citation: Campana I, Cinotti E, Broganelli P, Di Stefani A, Trovato E, Provvidenziale L, Perotti R, Lamberti A, Rubegni P. Neurothekeomas: dermoscopic features of 3 cases. Dermatol Pract Concept. 2019;9(4):322-324. DOI: https://doi.org/10.5826/dpc.0904a21

Accepted: July 3, 2019; Published: October 31, 2019

Copyright: (92019 Campana et al. This is an open-access article distributed under the terms of the Creative Commons Attribution License, which permits unrestricted use, distribution, and reproduction in any medium, provided the original author and source are credited.

Funding: None.

Competing interests: The authors have no conflicts of interest to disclose.

Authorship: All authors have contributed significantly to this publication.

Corresponding author: Irene Campana, MD, Department of Medical, Surgical and Neurological Science, Dermatology Section, University of Siena, S. Maria alle Scotte Hospital, Viale Bracci 16, 53100 Siena, Italy. Email: irenecamp89@gmail.com

\section{Introduction}

Pink lesions can be clinically challenging to diagnose, and dermoscopy can be useful to better characterize them. We describe clinical and dermoscopic features of 3 neurothekeomas, rare pink tumors of which only 2 dermoscopic descriptions are available.

\section{Case Presentation}

The first case concerned a 45-year-old woman affected by multiple sclerosis, who came to our observation for an asymptomatic nodule on her left forearm, slow-growing for 10 months.

Clinical examination showed a pinkish nodule of approximately $1 \mathrm{~cm}$ in size, fixed and soft at palpation, with a macroscopically detectable arborizing vessel (Figure 1A). Dermoscopy revealed pink ovoid areas in the center surrounded by a whitish halo on a yellowish background (Figure 1B). Histological examination showed a dermal multilobular unencapsulated mass, extending to subcutis, composed of spindle cells dispersed in a large amount of myxoid stroma, a description that allowed the diagnosis of mixed neurothekeoma (cellular and myxoid) (Figure 1C).

The second and third case corresponded to slow-growing asymptomatic nodular pinkish lesions, located on the right shoulder of a 58-year-old man (Figure 1D) and on the ala nasi of a 14-year-old girl (Figure 1G). Dermoscopy showed 2 flesh-colored lesions with irregular linear and arborizing thin and thick vessels (Figure 1, E and H). In both cases, histological examination was essential for the diagnosis of mixed neurothekeoma (Figure 1, F, I, and J).

\section{Discussion}

The term neurothekeoma was coined in 1980 by Gallagher and Helwig with reference to a rare dermal benign tumor originating from the sheath of peripheral nerves. Until now, histogenesis remains a tough question: neuroectodermal, fibrohistiocytic, and myofibroblastic derivation have been 


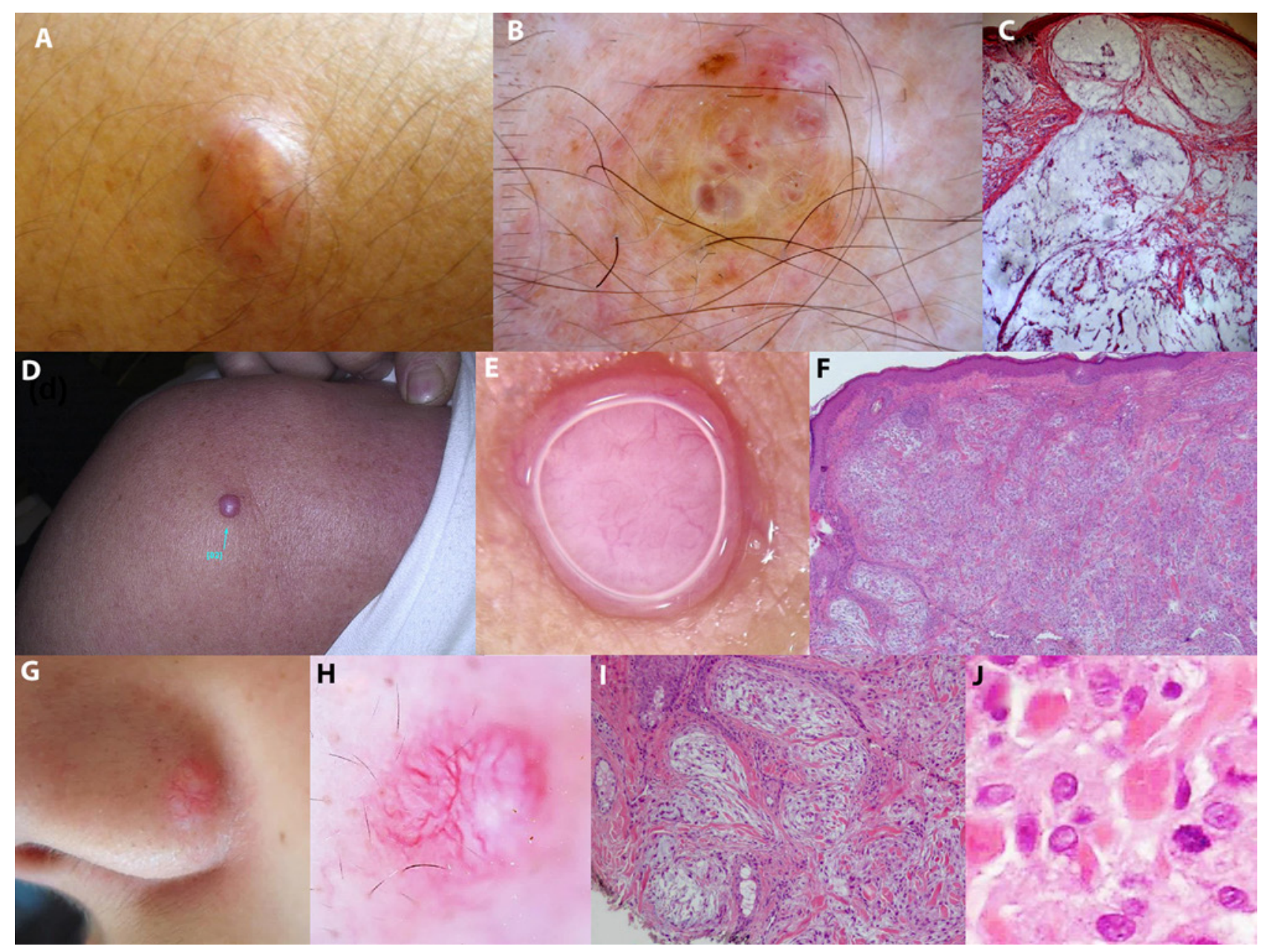

Figure 1. Clinical, dermoscopic, and histological features of 3 neurothekeomas. Case 1: (A) Pink nodule. (B) Dermoscopy shows pink ovoid areas in the center surrounded by a whitish halo and a yellowish background. (C) Histological examination (H\&E stain $\times 100)$ shows a mixed neurothekeoma: plexiform architecture in which each single nodule appears variable in size, separated from the others by fibrous septa and composed of areas of myxoid substance with proliferation of spindle cells at the periphery. Case 2: (D) Pink nodule. (E) Dermoscopy shows irregular linear and arborizing vessels on a homogeneous pink-whitish background. (F) Histological examination $(\mathrm{H} \& \mathrm{E} \times 50)$ shows a mixed type neurothekeoma: a poorly circumscribed lobular mass involving reticular dermis and subcutis, with small nests and fascicles of short spindle cells in a variable myxomatous stroma separated by thin fibrous septa which correspond to sclerotic collagen bundles. Case 3: (G) Pink nodule. (H) Dermoscopy reveals thick, well-focused arborizing vessels, irregular linear vessels, and some white areas on a dull pink background. (I) Histological examination $(\mathrm{H} \& \mathrm{E} \times 100)$ shows a mixed type neurothekeoma with nests and fascicles of spindle and epithelioid cells that sometimes have a concentric or whirling arrangement and a myxoid stroma; neoplastic cells $(\mathrm{J})(\mathrm{H} \& \mathrm{E} \times 400)$ are recognized for their pale eosinophilic cytoplasm, their vesicular nuclei, and their indistinct cell membranes. [Copyright: @2019 Campana et al.]

proposed. On the basis of the amount of myxoid matrix and the immunohistochemical pattern, 3 histopathological variants are recognized: cellular, myxoid, and mixed. Clinical diagnosis is challenging and neurothekeoma is often mistaken for basal cell carcinoma, achromic melanoma, Spitz nevus, or adnexal tumor (mainly pilomatricoma). Dermoscopic features of neurothekeoma have been previously reported in only 2 cases of myxoid [1] and cellular [2] variant. Both tumors were characterized by well-focused arborizing vessels, and one [1] was also connoted by a whitish area and white clods that were related to fibrotic septa and peripheral fibrosis of the tumor. Our cases confirmed that neurothekeoma should be included among nodular basal cell carcinoma mimickers because it can show arborizing vessels that are notoriously a hallmark of this tumor [2]. Moreover arborizing vessels can also be found in other amelanotic nodular skin tumors, such as Merkel cell carcinoma and adnexal tumors, which should also be considered among the differential diagnosis on dermoscopy. Our observation adds new dermoscopic findings to neurothekeoma that could represent integrating clues for its diagnosis: pinkish areas surrounded by a whitish halo and a yellowish background. The latter could be related to the amount of myxoid component; pinkish areas, instead, could reflect the peculiar lobular morphology of our tumor encircled by fibrosis. 


\section{Conclusions}

Neurothekeoma should be included in the maze of differential diagnoses of pink lesions and in particular among mimickers of basal cell carcinoma on dermoscopy. Nevertheless, further cases are needed to establish the diagnostic value of white areas and pinkish areas surrounded by a whitish halo and yellowish background.

\section{References}

1. Cavicchini S, Guanziroli E, Del Gobbo A, Scaparro M, Gianotti R. Neurothekeoma, a hard to diagnose neoplasm among red nodules. Australas J Dermatol. 2018;59(4):e280-e282.

2. Aydingoz IE, Mansur AT, Dikicioglu-Cetin E. Arborizing vessels under dermoscopy: a case of cellular neurothekeoma instead of basal cell carcinoma. Dermatology Online J. 2013;19(3):5. 